DOI: $10.15593 / 2224-9982 / 2021.64 .10$

УДК 519.9, 629.7

М.Ю. Егоров ${ }^{1}$, Д.М. Егоров ${ }^{2}$, С.М. Егоров ${ }^{2}$

${ }^{1}$ Пермский национальный исследовательский политехнический университет, Пермь, Россия

${ }^{2}$ Научно-исследовательский институт полимерных материалов, Пермь, Россия

\author{
ЧИСЛЕННОЕ ИССЛЕДОВАНИЕ ДИНАМИКИ ВНУТРИКАМЕРНЫХ \\ ПРОЦЕССОВ В МАРШЕВОМ РАКЕТНОМ ДВИГАТЕЛЕ НА ТВЕРДОМ \\ ТОПЛИВЕ С УЧЕТОМ ПОЛЕТНЫХ ПЕРЕГРУЗОК.
}

\author{
ЧАСТЬ 1. МЕТОДИКА РАСЧЕТА
}

\begin{abstract}
Исследуется динамика переходных внутрикамерных процессов (внутренняя баллистика) маршевого РДТТ второй ступени крылатой ракеты с учетом в общем случае распределенных пространственно-трехмерных и изменяющихся во времени полетных перегрузок. Метод исследования - постановка вычислительного эксперимента. Рассматривается сопряженная постановка задачи, включающая в себя: нестационарное срабатывание воспламенительного устройства (скорость горения воспламенительного состава описывается на основе экспериментально-теоретического подхода, учитывающего догорание продуктов сгорания за корпусом воспламенительного устройства); прогрев, зажигание и последующее нестационарное и турбулентное горение заряда твердого топлива (используется квазигомогенная модель горения на основе уравнений теплопроводности и химической кинетики, записанных для конденсированной фазы (твердого топлива), с учетом влияния газовой фазы (факела) на процесс горения в конденсированной фразе; метод решения задачи - метод конечных разностей); нестационарное трехмерное гомогенно-гетерогенное четырехфазное течение воздуха и продуктов сгорания в камере сгорания, сопловом блоке и за сопловым блоком ракетного двигателя (используются подходы механики сплошных многофазных сред; базовая система уравнений - система вихревых дифференциальных уравнений газовой динамики; метод решения - многопараметрический класс разностных схем расщепления по физическим процессам метода Давыдова); разгерметизацию камеры сгорания РДТТ (уравнение движения заглушки соплового блока - второй закон Ньютона; метод решения задачи - метод Эйлера). Каждая из подзадач рассматривается во взаимосвязи и разрешается одновременно - на одном шаге по времени. Для решения сформулированной задачи разработан комплекс прикладных программ с использованием (для основного расчетного модуля) стандарта многопотоковой обработки информации OpenCL. Проведена проверка работоспособности программного продукта.

Ключевые слова: численное исследование, ракетный двигатель твердого топлива, горение твердого топлива, газовая динамика, полетные перегрузки, методика расчета.
\end{abstract}

\author{
M.Yu. Egorov ${ }^{1}$, D.M. Egorov ${ }^{2}$, S.M. Egorov ${ }^{2}$ \\ ${ }^{1}$ Perm National Research Polytechnic University, Perm, Russian Federation \\ ${ }^{2}$ Research Institute of Polymer Materials, Perm, Russian Federation
}

\title{
NUMERICAL STUDY OF DYNAMICS INTRACHAMBER PROCESSES IN SOLID PROPELLANT SUSTAINER TAKING INTO ACCOUNT FLIGHT OVERLOADS. \\ PART 1. CALCULATION METHOD
}

The dynamics of transient in-chamber processes (internal ballistics) of the cruise missile's second-stage cruise missile propulsion system is studied, taking into account, in the general case, distributed spatially-three-dimensional and time-varying flight overloads. The research method is the formulation of a computational experiment. Be considered coupled formulation of the problem, including: - transient triggering of igniter device (the rate of combustion of the igniting composition is described on the basis of experimental and theoretical approach afterburn combustion products in case igniting device); preheating, ignition and subsequent unsteady and turbulent combustion of solid propellant charge (used quasi-homogeneous combustion model based on the equations of heat conduction and chemical kinetics recorded for a condensed phase (solid fuel), taking into account the influence of the gas phase (torch) on the process of combustion in the condensed phase; the method of solving the problem - finite difference method); - non-stationary three-dimensional homogeneous-heterogeneous four phase flow of air and products of combustion in the combustion chamber, the nozzle block and the block launchers rocket engine (used approaches of continuum mechanics of multiphase media; the basic system of equations system of vortex differential equations of gas dynamics solution method - a multi-parameter class of difference schemes of splitting into physical processes of the method Davydova); - depressurization of the combustion chamber of the SRB (equation of motion of the plug nozzle block - Newton's second law; the proposed solution method - Euler's 
method). Each of the subtasks is considered in a relationship and resolved simultaneously - at one time step. To solve the formulated problem, a set of application programs has been developed using (for the main calculation module) the OpenCL multithreaded information processing standard. The performance of the software product was checked.

Keywords: numerical study, solid fuel rocket engine, burning of solid propellant, gas dynamics, flight overloads, calculation method.

\section{Введение}

Численное исследование переходных внутрикамерных процессов (так называемой внутренней баллистики) производится на этапе проектирования РДТТ [1-7]. Исследуются особенности процесса срабатывания воспламенительного устройства, прогрев, зажигание и горение основного заряда твердого топлива, газовая динамика в камере сгорания, в сопловом блоке, за сопловым блоком и другие вспомогательные задачи. Далее осуществляется конструирование ракетного двигателя, изготовление опытной партии РДТТ и его стендовая отработка. Результаты численных расчетов сопоставляются с данными стендовых испытаний РДТТ. После этого разработанное изделие (ракетный двигатель) в составе ракетного комплекса переходит на этап летных испытаний. Очевидно, что условия летных испытаний не в полной мере соответствуют условиям проектирования и стендовой отработки РДТТ, особенно тогда, когда величина планируемых полетных перегрузок значительная (более $10 \mathrm{~g}$ ). В этом случае у разработчиков ракетной техники возникают вопросы, связанные как минимум с оценкой влияния перегрузок и как максимум с полным анализом влияния внешней аэродинамики и поля силы тяжести на внутрикамерные процессы и, соответственно, на выходные характеристики РДТТ (расход продуктов сгорания, тяга двигателя, время релаксации камеры сгорания и пр.).

Ранее в ряде работ одного из авторов статьи проводились исследования влияния осевой полетной перегрузки на динамику волнового процесса в камере сгорания РДТТ [8, 9 и др.].
Было установлено, что значительные осевые перегрузки влияют на амплитуду колебаний давления (по первой продольной моде) в камере сгорания. По мере увеличения уровня перегрузки наблюдается уменьшение амплитуды колебаний давления в камере сгорания и амплитуды колебаний тяги ракетного двигателя, хотя общий характер силового нагружения ракетной системы при перегрузках усложняется.

Предлагается продолжить исследование в этом важном для теории и практики направлении. Используя современные возможности вычислительной математики (в первую очередь численных методов), методов алгоритмирования и программирования, методов обработки информации и высокопроизводительных вычислительных систем, можно перейти на более глубокий уровень понимания динамики рассматриваемых процессов.

Для этого в данной работе исследуется динамика переходных внутрикамерных процессов (внутренняя баллистика) маршевого РДТТ второй ступени крылатой ракеты с учетом распределенных пространственно-трехмерных и изменяющихся во времени полетных перегрузок. Принципиальная компоновочная схема ракетного двигателя показана на рис. 1.

Метод исследования - постановка прямого вычислительного эксперимента (основной расчетный метод - метод Давыдова). Рассматривается сопряженная постановка задачи, включающая в себя: нестационарное срабатывание воспламенительного устройства; прогрев, зажигание и последующее нестационарное и турбулентное горение заряда твердого топлива; нестационарное трехмерное гомогенно-гетерогенное четырехфазное течение

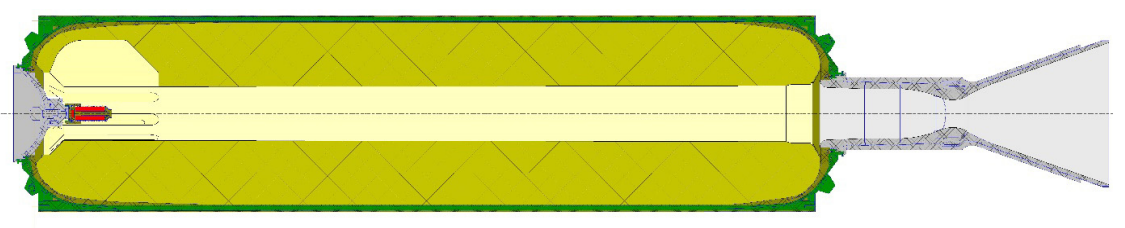

Рис. 1. Компоновочная схема РДТТ 
воздуха и продуктов сгорания в камере сгорания, сопловом блоке и за сопловым блоком ракетного двигателя; разгерметизацию камеры сгорания РДТТ (вскрытие заглушки соплового блока). Каждая из подзадач рассматривается во взаимосвязи и разрешается одновременно на одном шаге по времени.

\section{Срабатывание воспламенительного устройства}

Процесс срабатывания воспламенительного устройства (ВУ) с жестким несгораемым перфорированным корпусом и вкладным формованным зарядом пиротехнического воспламенительного состава описывается на основе экспериментально-теоретического подхода. Путем решения обратной задачи внутренней баллистики для системы «ВУ - имитатор свободного объема камеры сгорания РДТТ» рассчитывается реальный газоприход от ВУ с учетом особенности горения заряда пиротехнического воспламенительного состава - догорания продуктов сгорания за корпусом ВУ. Скорость горения заряда ВУ $v_{\text {в }}$ напрямую связывается с параметрами, определяющими процесс горения в корпусе ВУ (площадью перфорации корпуса ВУ $F_{\text {кр }}$, площадью поверхности горения заряда ВУ $S_{\text {в }}$ и свободным объемом в корпусе ВУ $\left.V_{\text {в }}\right)$, в виде зависимости $v_{\text {в }}=f\left(F_{\text {кр }}, S_{\text {в }}, V_{\text {в }}\right)$.

Такого рода связи, не вникая в сущность сложного физико-химического процесса горения, можно найти в статистическом подходе, используя полиномиальные модели вида

$$
\begin{gathered}
v_{\text {в }}=A_{0}+A_{1} X 1+A_{2} X 2+A_{3} X 1 X 2+ \\
+A_{4} X 1^{2}+A_{5} X 2^{2}+A_{6} X 1 X 2^{2} ; \\
X 1=\left(\frac{F_{\text {кр }}}{V_{\text {в }}}-B_{1}\right) / B_{2} ; \quad X 2=\left(\frac{S_{\text {в }}}{V_{\text {в }}}-C_{1}\right) / C_{2},
\end{gathered}
$$

a для нахождения коэффициентов полинома применить теорию планирования эксперимента. Здесь $A_{0}, \ldots, A_{6}, B_{1}, B_{2}, C_{1}, C_{2}$ - коэффициенты полинома, зависящие от конкретного вида пиротехнического воспламенительного состава и интервалов варьирования конструктивных параметров ВУ. Более подробно методика расчета изложена в работе [10] и других работах.
По полученной скорости горения заряда пиротехнического воспламенительного состава (1) определяется газоприход от ВУ в камеру сгорания РДТТ.

\section{Прогрев, зажигание и горение заряда твердого топлива}

Описание процесса нестационарного прогрева, воспламенения и последующего нестационарного и турбулентного горения заряда смесевого твердого топлива базируется на модели Мержанова - Дубовицкого-Соркина с учетом, в рамках подхода Горохова - Липанова Русяка, влияния газовой фазы на процесс горения в конденсированной фазе ( $k$-фазе) $[1,3,4]$. Используется так называемая квазигомогенная модель горения твердого топлива.

Будем рассматривать твердое топливо как твердое тело, к которому применимы известные уравнения теплопроводности и химической кинетики. Для удобства будем рассматривать эти уравнения в системе координат, связанной с поверхностью горения, направив ось от поверхности вглубь пороха. Считаем, что реакции в $k$-фазе удовлетворяют закону Аррениуса. Тогда в предположении 0-мерности порядка химических реакций данная система уравнений, описывающая процесс, имеет вид

$$
\begin{gathered}
\frac{\partial T_{k}}{\partial t}=\kappa_{k} \frac{\partial^{2} T_{k}}{\partial y_{k}^{2}}+v_{k} \frac{\partial T_{k}}{\partial y_{k}}+\frac{Q_{k}}{c_{k}} \Phi_{k}\left(T_{k}\right) ; \\
\frac{\partial \beta}{\partial t}=v_{k} \frac{\partial \beta}{\partial y_{k}}+\Phi_{k}\left(T_{k}\right),
\end{gathered}
$$

где $\Phi_{k}\left(T_{k}\right)=Z_{k} \exp \left(-\frac{E_{k}}{R_{0} T_{k}}\right)$.

До воспламенения необходимо положить $v_{k}=0$. Условие воспламенения и последующего горения: $\beta_{s}=\beta_{*}=1$.

До воспламенения начальные и граничные условия для системы (2) имеют вид

$$
\begin{aligned}
& t=0, \quad y_{k} \geq 0, \quad T_{k}=T_{0}, \quad \beta=0 \\
& t \geq 0, \quad y_{k}=0, \quad-\lambda_{k} \frac{\partial T_{k}}{\partial y_{k}}=\alpha_{\tau}\left(T_{g}-T_{s}\right), \quad \beta<\beta_{*} \\
& t \geq 0, \quad y_{k}=\infty, \quad T_{k}=T_{0}, \quad \beta=0 .
\end{aligned}
$$

После воспламенения граничные условия запишутся в виде 


$$
\begin{aligned}
& t>t_{*}, \quad y_{k}=0, \quad T_{k}=T_{s}, \quad \beta_{s}=\beta_{*}, \\
& -\lambda_{k} \frac{\partial T_{k}}{\partial y_{k}}=q_{s}-\left(c_{p s}-c_{k}\right) \rho_{k} v_{k} T_{s}-\left(a-\frac{1}{\rho_{k}}\right) \rho_{k} v_{k} p \\
& t>t_{*}, \quad y_{k}=\infty, \quad T_{k}=T_{0}, \quad \beta=0 .
\end{aligned}
$$

Задача (2)-(4), дополненная замыкающими соотношениями, обезразмеренная и записанная в неравномерной (экспоненциальной) системе координат, интегрируется численным сеточным методом по явным и неявным конечноразностным схемам $[4,11]$. Неявные схемы разрешаются способом прогонки.

Температура по своду $k$-фазы, исключая поверхность горения пороха,

$$
T_{i}^{j+1}=A_{i} T_{i+1}^{j+1}+B_{i} ; \quad 2 \leq i \leq(M-1) .
$$

Температура на поверхности горения пороха:

- до воспламенения

$$
T_{1}^{j+1}=A_{1} T_{2}^{j+1}+B_{1} ;
$$

- после воспламенения

$$
T_{1}^{j+1}=A_{1^{*}} T_{2}^{j+1}+B_{1^{*}} .
$$

Глубина превращения пороха:

- до воспламенения

$$
\beta_{i}^{j+1}=\beta_{i}^{j}+\Delta t a_{3} \Psi_{i}^{j}, \quad 1 \leq i \leq(M-1) ;
$$

- после воспламенения

$$
\beta_{i}^{j+1}=A_{i^{*} *} \beta_{i+1}^{j+1}+B_{i^{* *}}, \quad 1 \leq i \leq(M-1) .
$$

Скорость горения порохового заряда $v_{k}$ определяется из формулы (9) с учетом граничных условий $\beta_{1}^{j+1}=\beta_{1}^{j}=\beta_{*}, \quad \beta_{M}^{j+1}=\beta_{M}^{j}=0$ итерационным способом (методом секущих).

В формулах (2)-(9) и далее по тексту приняты обозначения: $T$ - температура, $t-$ время, $\kappa-$ коэффициент температуропроводности, $y$ - координата, $Q$ - тепловой эффект реакции, $c$ - удельная теплоемкость, $\beta$ - глубина превращения пороха, $Z$ - предэкспонент, $E$ - энергия активации, $R_{0}$ - универсальная газовая постоянная, $\lambda-$ коэффициент теплопроводности, $\alpha_{\tau}-$ коэффициент теплоотдачи, $q$ - плотность теплового потока, $a$ - коволюм, $A, B$ - коэффициенты прогонки, $\Delta t$ - шаг по времени, $a_{3}$ - const, $\Psi$ - безразмерная экспо- ненциальная функция температуры. Символы: $k$ - конденсированная фаза (твердое топливо), $g$ - газ, $s$ - поверхность горения, $p$ - давление, $i$ - номер расчетной точки по координате, $j-$ номер расчетной точки по времени, * - специальное значение.

По полученной скорости горения определяется газоприход от поверхности горения заряда твердого топлива в камеру сгорания РДТТ.

\section{Газовая динамика в камере сгорания РДТТ}

Для математического описания процесса течения в камере сгорания, сопловом блоке и за сопловым блоком РДТТ используем подходы механики сплошных многофазных сред, предложенные в работах Х.А. Рахматулина и Р.И. Нигматулина [12, 13]. Согласно этим подходам воздух, газообразные продукты сгорания пиротехнического воспламенительного состава и заряда смесевого твердого топлива назовем первой фазой, мелкодисперсные несгоревшие частицы (жидкие капли) в продуктах сгорания пиротехнического воспламенительного состава - второй фазой, мелкодисперсные несгоревшие частицы (жидкие капли) малого диаметра в продуктах сгорания заряда смесевого твердого топлива - третьей фазой, мелкодисперсные несгоревшие частицы (жидкие капли) большого диаметра в продуктах сгорания заряда смесевого твердого топлива четвертой фазой. Первую, вторую, третью и четвертую фазы будем считать гомогенногетерогенной смесью со своими температурами и скоростями движения. В такой системе каждая фаза занимает часть объема смеси: $\alpha_{1}, \alpha_{2}, \alpha_{3}, \alpha_{4}$. Здесь $\alpha_{i}=\rho_{i} / \rho_{i}^{\text {и }} \quad(i=1 \ldots 4)$, где $\rho_{i}^{\text {и }}$ - истинная плотность фазы. Движение этих фаз рассматривается как движение взаимопроникающих и взаимодействующих сред.

Тогда полная (нестационарная и трехмерная) система вихревых дифференциальных уравнений газовой динамики для сложного гомогенно-гетерогенного потока в камере сгорания, сопловом блоке и за сопловым блоком ракетного двигателя запишется в виде:

- уравнения неразрывности (сохранения массы) 


$$
\begin{gathered}
\frac{\partial \rho_{1}}{\partial t}+\operatorname{div}\left(\rho_{1} \mathbf{W}_{1}\right)=G_{g \mathrm{~B}}+G_{g w} \\
\frac{\partial \rho_{2}}{\partial t}+\operatorname{div}\left(\rho_{2} \mathbf{W}_{2}\right)=G_{p 2 \mathrm{~B}} \\
\frac{\partial \rho_{3}}{\partial t}+\operatorname{div}\left(\rho_{3} \mathbf{W}_{3}\right)=G_{p 3 w} \\
\frac{\partial \rho_{4}}{\partial t}+\operatorname{div}\left(\rho_{4} \mathbf{W}_{4}\right)=G_{p 4 w} \\
\frac{\partial\left(\rho_{1} \varphi\right)}{\partial t}+\operatorname{div}\left(\rho_{1} \varphi \mathbf{W}_{1}\right)=\varphi_{\mathrm{B}} G_{g \mathrm{~B}}+\varphi_{w} G_{g w}, \\
\varphi=k, c_{\mathrm{p}}, \mu, \lambda, a
\end{gathered}
$$
осям координат

- уравнения сохранения импульса по

$$
\begin{aligned}
& \frac{\partial\left(\rho_{1} u_{1}\right)}{\partial t}+\operatorname{div}\left(\rho_{1} u_{1} \mathbf{W}_{1}\right)+\alpha_{1} \frac{\partial p}{\partial x}= \\
& =\rho_{1} N_{x} g-\tau_{x}^{12}-\tau_{x}^{13}-\tau_{x}^{14}+u_{\mathrm{B}} G_{g_{\mathrm{B}}}+u_{w} G_{g w} ; \\
& \frac{\partial\left(\rho_{1} v_{1}\right)}{\partial t}+\operatorname{div}\left(\rho_{1} v_{1} \mathbf{W}_{1}\right)+\alpha_{1} \frac{\partial p}{\partial y}= \\
& =\rho_{1} N_{y} g-\tau_{y}^{12}-\tau_{y}^{13}-\tau_{y}^{14}+v_{\mathrm{B}} G_{g_{\mathrm{B}}}+v_{w} G_{g w} ; \\
& \frac{\partial\left(\rho_{1} w_{1}\right)}{\partial t}+\operatorname{div}\left(\rho_{1} w_{1} \mathbf{W}_{1}\right)+\alpha_{1} \frac{\partial p}{\partial z}= \\
& =\rho_{1} N_{z} g-\tau_{z}^{12}-\tau_{z}^{13}-\tau_{z}^{14}+w_{\mathrm{B}} G_{g_{\mathrm{B}}}+w_{w} G_{g w} ; \\
& \frac{\partial\left(\rho_{2} u_{2}\right)}{\partial t}+\operatorname{div}\left(\rho_{2} u_{2} \mathbf{W}_{2}\right)+\alpha_{2} \frac{\partial p}{\partial x}= \\
& =\rho_{2} N_{x} g+\tau_{x}^{12}+u_{\mathrm{B}} G_{p 2 \mathrm{~B}} \text {; } \\
& \frac{\partial\left(\rho_{2} v_{2}\right)}{\partial t}+\operatorname{div}\left(\rho_{2} v_{2} \mathbf{W}_{2}\right)+\alpha_{2} \frac{\partial p}{\partial y}= \\
& =\rho_{2} N_{y} g+\tau_{y}^{12}+v_{\mathrm{B}} G_{p 2 \mathrm{~B}} \text {; } \\
& \frac{\partial\left(\rho_{2} w_{2}\right)}{\partial t}+\operatorname{div}\left(\rho_{2} w_{2} \mathbf{W}_{2}\right)+\alpha_{2} \frac{\partial p}{\partial z}= \\
& =\rho_{2} N_{z} g+\tau_{z}^{12}+w_{\mathrm{B}} G_{p 2 \mathrm{~B}} \text {; } \\
& \frac{\partial\left(\rho_{3} u_{3}\right)}{\partial t}+\operatorname{div}\left(\rho_{3} u_{3} \mathbf{W}_{3}\right)+\alpha_{3} \frac{\partial p}{\partial x}= \\
& =\rho_{3} N_{x} g+\tau_{x}^{13}+u_{w} G_{p 3 w} ; \\
& \frac{\partial\left(\rho_{3} v_{3}\right)}{\partial t}+\operatorname{div}\left(\rho_{3} v_{3} \mathbf{W}_{3}\right)+\alpha_{3} \frac{\partial p}{\partial y}= \\
& =\rho_{3} N_{y} g+\tau_{y}^{13}+v_{w} G_{p 3 w} ; \\
& \frac{\partial\left(\rho_{3} w_{3}\right)}{\partial t}+\operatorname{div}\left(\rho_{3} w_{3} \mathbf{W}_{3}\right)+\alpha_{3} \frac{\partial p}{\partial z}= \\
& =\rho_{3} N_{z} g+\tau_{z}^{13}+w_{w} G_{p 3 w} ;
\end{aligned}
$$

$$
\begin{aligned}
& \frac{\partial\left(\rho_{4} u_{4}\right)}{\partial t}+\operatorname{div}\left(\rho_{4} u_{4} \mathbf{W}_{4}\right)+\alpha_{4} \frac{\partial p}{\partial x}= \\
& =\rho_{4} N_{x} g+\tau_{x}^{14}+u_{w} G_{p 4 w} ; \\
& \frac{\partial\left(\rho_{4} v_{4}\right)}{\partial t}+\operatorname{div}\left(\rho_{4} v_{4} \mathbf{W}_{4}\right)+\alpha_{4} \frac{\partial p}{\partial y}= \\
& =\rho_{4} N_{y} g+\tau_{y}^{14}+v_{w} G_{p 4 w} ; \\
& \frac{\partial\left(\rho_{4} w_{4}\right)}{\partial t}+\operatorname{div}\left(\rho_{4} w_{4} \mathbf{W}_{4}\right)+\alpha_{4} \frac{\partial p}{\partial z}= \\
& =\rho_{4} N_{z} g+\tau_{z}^{14}+w_{w} G_{p 4 w} ;
\end{aligned}
$$

- уравнения сохранения внутренней удельной энергии второй, третьей и четвертой фазы

$$
\begin{aligned}
& \frac{\partial\left(\rho_{2} J_{2}\right)}{\partial t}+\operatorname{div}\left(\rho_{2} J_{2} \mathbf{W}_{2}\right)=q_{k}^{12}+q_{l}^{12}+J_{p 2 \mathrm{~B}} G_{p 2 \mathrm{~B}} ; \\
& \frac{\partial\left(\rho_{3} J_{3}\right)}{\partial t}+\operatorname{div}\left(\rho_{3} J_{3} \mathbf{W}_{3}\right)=q_{k}^{13}+q_{l}^{13}+J_{p 3 w} G_{p 3 w} ; \\
& \frac{\partial\left(\rho_{4} J_{4}\right)}{\partial t}+\operatorname{div}\left(\rho_{4} J_{4} \mathbf{W}_{4}\right)=q_{k}^{14}+q_{l}^{14}+J_{p 4 w} G_{p 4 w} ;
\end{aligned}
$$

- уравнения сохранения полной удельной энергии смеси

$$
\begin{aligned}
& \frac{\partial\left(\rho_{1} E_{1}\right)}{\partial t}+\frac{\partial\left(\rho_{2} E_{2}\right)}{\partial t}+\frac{\partial\left(\rho_{3} E_{3}\right)}{\partial t}+\frac{\partial\left(\rho_{4} E_{4}\right)}{\partial t}+ \\
& +\operatorname{div}\left(\rho_{1} E_{1} \mathbf{W}_{1}\right)+\operatorname{div}\left(\rho_{2} E_{2} \mathbf{W}_{2}\right)+\operatorname{div}\left(\rho_{3} E_{3} \mathbf{W}_{3}\right)+ \\
& +\operatorname{div}\left(\rho_{4} E_{4} \mathbf{W}_{4}\right)+\operatorname{div}\left(\alpha_{1} p \mathbf{W}_{1}\right)+\operatorname{div}\left(\alpha_{2} p \mathbf{W}_{2}\right)+ \\
& +\operatorname{div}\left(\alpha_{3} p \mathbf{W}_{3}\right)+\operatorname{div}\left(\alpha_{4} p \mathbf{W}_{4}\right)=N_{x} g \sum_{i=1}^{i=4}\left(\rho_{i} u_{i}\right)+ \\
& +N_{y} g \sum_{i=1}^{i=4}\left(\rho_{i} v_{i}\right)+N_{z} g \sum_{i=1}^{i=4}\left(\rho_{i} w_{i}\right)+E_{g \mathrm{~B}} G_{g \mathrm{~B}}+ \\
& +E_{g w} G_{g w}+E_{p 2 \mathrm{~B}} G_{p 2 \mathrm{~B}}+E_{p 3 w} G_{p 3 w}+E_{p 4 w} G_{p 4 w}
\end{aligned}
$$

где для декартовой системы координат

$$
\begin{aligned}
& \operatorname{div}\left(\xi \mathbf{W}_{i}\right)=\frac{\partial\left(\xi u_{i}\right)}{\partial x}+\frac{\partial\left(\xi v_{i}\right)}{\partial y}+\frac{\partial(\xi w)}{\partial z}, \\
& \xi=\left[\rho_{i}, \rho_{1} \varphi, \rho_{i} u_{i}, \rho_{i} v_{i}, \rho_{i} w_{i}, \rho_{j} J_{j}, \rho_{i} E_{i}, \alpha_{i} p\right] \\
& i=(1 \ldots 4) ; j=(2 \ldots 4) .
\end{aligned}
$$

Для замыкания системы дифференциальных уравнений (10)-(14) используем уравнение состояния в виде

$$
p=(k-1) \rho_{1}^{\mathrm{u}}\left(E_{1}-\frac{W_{1}^{2}}{2}\right) \frac{1}{1-a \rho_{1}^{\mathrm{u}}} .
$$


В уравнениях (10)-(15) и далее по тексту приняты обозначения: $c_{\mathrm{p}}-$ удельная теплоемкость при постоянном давлении, $E$ - полная удельная энергия, $J$ - внутренняя удельная энергия, $G$ - расходно-приходный комплекс, $g$ - ускорение свободного падения, $k$ - показатель адиабаты, $N$ - осевая перегрузка гравитационного поля силы тяжести, $q$ - функция теплового межфазного взаимодействия; $u, v, w-$ проекции вектора скорости по осям координат $0 X, 0 Y, 0 Z ; \mathbf{W}$ - вектор скорости, $W$ - модуль вектора скорости; $x, y, z$ - текущие координаты вдоль осей $0 X, 0 Y, 0 Z ; \mu$ - динамическая вязкость, $\tau$ - функция силового межфазного взаимодействия. Символы: $g$ - газ, $k$ - конвективный, $l$ - лучистый, $p$ - частицы, $w$ - заряд твердого топлива, $x$ - вдоль оси $0 X, y$ - вдоль оси $0 Y, z$ - вдоль оси $0 Z$.

Выражения (10)-(13) для расходно-приходных комплексов, функций силового и теплового межфазного взаимодействия, а также используемые в расчетах дополнительные соотношения подробно изложены в работе [4].

Система уравнений (10)-(15) с учетом дополнительных соотношений интегрируется численно с помощью метода Давыдова (метода крупных частиц) - метода постановки вычислительного эксперимента $[3,4,8-10,14-18]$. Область интегрирования покрывается фиксированной в трехмерном пространстве (эйлеровой) равномерной (однородной и полностью изотропной) кубической расчетной сеткой с ячейками $\Delta x \times \Delta y \times \Delta z$. Для такой сетки геометрический центр ячейки совпадает с центром масс ячейки, что весьма существенно для получения точного результата. Значения целых чисел $i$ (вдоль оси $0 X), j$ (вдоль оси 0Y) и $k$ (вдоль оси $0 Z)$ обозначают геометрический центр ячейки, $n$ - порядковый номер шага по времени.

Эйлеров этап метода. На этом этапе расчета изменяются величины, относящиеся к ячейке в целом, а исследуемая среда предполагается заторможенной (на этом этапе также учитывается вклад гравитационного поля силы тяжести):

$$
\begin{gathered}
\tilde{u}_{l_{i, j, k}}^{n}=u_{l_{i, j, k}}^{n}-\alpha_{l_{i, j, k}}^{n} \times \\
\times \frac{p_{i+0,5, j, k}^{n}-p_{i-0,5, j, k}^{n}}{\Delta x} \frac{\Delta t}{\rho_{l_{i, j, k}}^{n}}+N_{x} g \Delta t ; \quad l=1 . . .4 ;
\end{gathered}
$$

$$
\tilde{E}_{m_{i, j, k}}^{n}=J_{m_{i, j, k}}^{n}+\frac{\left(\tilde{u}_{m_{i, j, k}}^{n}\right)^{2}+\left(\tilde{v}_{m_{i, j, k}}^{n}\right)^{2}}{2}, \quad m=2 \ldots 4
$$

аналогично по другим направлениям $\tilde{v}_{i, j, k}^{n}, \tilde{w}_{l_{i, j, k}}^{n} ;(16)$

$$
\tilde{E}_{1_{i, j, k}}^{n}=E_{1_{i, j, k}}^{n}-\sum_{m=2}^{m=4}\left[\left(\tilde{E}_{m_{i, j, k}}^{n}-E_{m_{i, j, k}}^{n}\right) \frac{\rho_{m_{i, j, k}}^{n}}{\rho_{1_{i, j, k}}^{n}}\right]-
$$

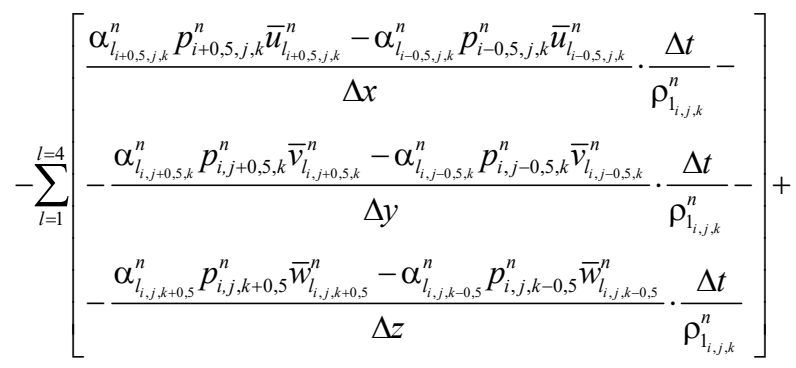

$$
\begin{aligned}
& +N_{x} g \sum_{l=1}^{l=4}\left(\rho_{l_{i, j, k}}^{n} u_{l_{i, j, k}}^{n}\right) \frac{\Delta t}{\rho_{i_{i, j, k}}^{n}}+ \\
& +N_{y} g \sum_{l=1}^{l=4}\left(\rho_{l_{i, j, k}}^{n} v_{l_{i, j, k}}^{n}\right) \frac{\Delta t}{\rho_{i_{i, j, k}}^{n}}+ \\
& +N_{z} g \sum_{l=1}^{l=4}\left(\rho_{l_{i, j, k}}^{n} w_{l_{i, j}}^{n}\right) \frac{\Delta t}{\rho_{1_{i, j, k}}^{n}},
\end{aligned}
$$

где $p_{i+0,5, j, k}^{n}=\frac{p_{i, j, k}^{n}+p_{i+1, j, k}^{n}}{2} ; \quad \bar{u}_{l_{i, j, k}^{n}}^{n}=(1-\mathrm{alfa}) \times$ $\times u_{l_{i, j, k}}^{n}+\mathrm{alfa} \cdot \tilde{u}_{l_{i, j, k}}^{n}$ и т.д. В формулах (16) alfaсеточный параметр, значение которого изменяется в пределах 1,0-3,0.

Лагранжев этап метода. На данном этапе метода вычисляются эффекты переноса (потоковые комплексы), учитывающие обмен между ячейками при их перестройке на прежнюю эйлерову сетку:

- первая фаза вдоль оси $0 X$

$$
\begin{aligned}
& \left(\rho_{1} \xi \tilde{u}_{1}\right)_{i+0,5, j, k}^{n}= \\
& \int\left[(1-\text { beta }) \rho_{1_{i, j, k}}^{n}+\text { beta } \cdot \rho_{1_{i+1, j, k}}^{n}\right] \xi_{i, j, k}^{n} \tilde{u}_{1_{i+0,5, j, k}}^{n}, \\
& = \begin{cases}\text { если } & \tilde{u}_{1_{i+0,5, j, k}^{n}}^{n} \geq 0 ;\end{cases}
\end{aligned}
$$

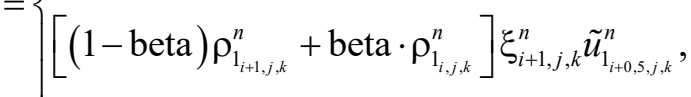

$$
\begin{aligned}
& \text { если } \tilde{u}_{1_{i+0,5, j, k}}^{n}<0 \text {; } \\
& \xi=\left(1, k, c_{\mathrm{p}}, \mu, \lambda, a, \tilde{u}_{1}, \tilde{v}_{1}, \tilde{w}_{1}, \tilde{E}_{1}\right) \text {; }
\end{aligned}
$$


аналогично по другим направлениям $\left(\rho_{1} \xi \tilde{v}_{1}\right)_{i, j+0,5, k}^{n},\left(\rho_{1} \xi \tilde{w}_{1}\right)_{i, j, k+0,5}^{n} ;$

- вторая, третья и четвертая фазы вдоль оси $0 X$

$$
\begin{gathered}
\left(\rho_{m} \xi \tilde{u}_{m}\right)_{i+0,5, j, k}^{n}=\left\{\begin{array}{l}
\rho_{m_{i, j, k}}^{n} \xi_{i, j, k}^{n} \tilde{u}_{m_{i+0,5, j, k}}^{n}, \\
\text { если } \quad \tilde{u}_{m_{i+0,5, j, k}} \geq 0 ; \\
\rho_{m_{i+1, j, k}}^{n} \xi_{i+1, j, k}^{n} \tilde{u}_{m_{i+0,5, j, k}}^{n}, \\
\text { если } \quad \tilde{u}_{m_{i+0,5, j, k}}^{n}<0 ;
\end{array}\right. \\
\xi=\left(1, \tilde{u}_{m}, \tilde{v}_{m}, \tilde{w}_{m}, J_{m}, \tilde{E}_{m}\right), \quad m=2 . .4
\end{gathered}
$$

аналогично по другим направлениям $\left(\rho_{m} \xi \tilde{v}_{m}\right)_{i, j+0,5, k}^{n},\left(\rho_{m} \xi \tilde{w}_{m}\right)_{i, j, k+0,5}^{n}$ и т.д. В формулах (17) beta - сеточный параметр, значение которого изменяется в пределах $-0,2-0,0$.

На лагранжевом этапе метода вычисляются также приходные комплексы и функции силового и теплового межфазного взаимодействия, входящие в уравнения (10)-(13), с учетом изменения параметров потока на эйлеровом этапе.

Заключительный этап метода. Здесь происходит перераспределение массы, импульса и энергии по пространству и определяются окончательные поля эйлеровых параметров потока на фиксированной сетке в новый момент времени:

- уравнения неразрывности (сохранения массы)

$$
\begin{aligned}
& \rho_{1_{i, j, k}}^{n+1}=\rho_{1_{i, j, k}}^{n}-\frac{\left(\rho_{1} \tilde{u}_{1}\right)_{i+0,5, j, k}^{n}-\left(\rho_{1} \tilde{u}_{1}\right)_{i-0,5, j, k}^{n}}{\Delta x} \Delta t- \\
& -\frac{\left(\rho_{1} \tilde{v}_{1}\right)_{i, j+0,5, k}^{n}-\left(\rho_{1} \tilde{v}_{1}\right)_{i, j-0,5, k}^{n}}{\Delta y} \Delta t- \\
& -\frac{\left(\rho_{1} \tilde{w}_{1}\right)_{i, j, k+0,5}^{n}-\left(\rho_{1} \tilde{w}_{1}\right)_{i, j, k-0,5}^{n}}{\Delta z} \Delta t+\left(G_{g_{\mathrm{B}_{i, j, k}}^{n}}^{n}+G_{g w_{i, j, k}}^{n}\right) \Delta t ;
\end{aligned}
$$$$
\rho_{2_{i, j, k}^{n+1}}^{n+1} \rho_{2_{i, j, k}}^{n}-\frac{\left(\rho_{2} \tilde{u}_{2}\right)_{i+0,5, j, k}^{n}-\left(\rho_{2} \tilde{u}_{2}\right)_{i-0,5, j, k}^{n}}{\Delta x} \Delta t-
$$$$
-\frac{\left(\rho_{2} \tilde{v}_{2}\right)_{i, j+0,5, k}^{n}-\left(\rho_{2} \tilde{v}_{2}\right)_{i, j-0,5, k}^{n}}{\Delta y} \Delta t-
$$

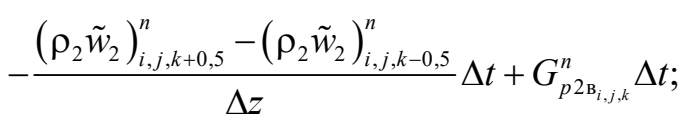

$$
\begin{aligned}
& \rho_{3_{i, j, k}}^{n+1}=\rho_{3_{i, j, k}}^{n}-\frac{\left(\rho_{3} \tilde{u}_{3}\right)_{i+0,5, j, k}^{n}-\left(\rho_{3} \tilde{u}_{3}\right)_{i-0,5, j, k}^{n}}{\Delta x} \Delta t- \\
& -\frac{\left(\rho_{3} \tilde{v}_{3}\right)_{i, j+0,5, k}^{n}-\left(\rho_{3} \tilde{v}_{3}\right)_{i, j-0,5, k}^{n}}{\Delta y} \Delta t- \\
& -\frac{\left(\rho_{3} \tilde{w}_{3}\right)_{i, j, k+0,5}^{n}-\left(\rho_{3} \tilde{w}_{3}\right)_{i, j, k-0,5}^{n}}{\Delta z} \Delta t+G_{p 3 w_{i, j, k}}^{n} \Delta t ; \\
& \rho_{4_{i, j, k}}^{n+1}=\rho_{4_{i, j, k}}^{n}-\frac{\left(\rho_{4} \tilde{u}_{4}\right)_{i+0,5, j, k}^{n}-\left(\rho_{4} \tilde{u}_{4}\right)_{i-0,5, j, k}^{n} \Delta t-}{\Delta x} \Delta x \\
& -\frac{\left(\rho_{4} \tilde{v}_{4}\right)_{i, j+0,5, k}^{n}-\left(\rho_{4} \tilde{v}_{4}\right)_{i, j-0,5, k}^{n} \Delta t-}{\Delta y}
\end{aligned}
$$

$$
-\frac{\left(\rho_{4} \tilde{w}_{4}\right)_{i, j, k+0,5}^{n}-\left(\rho_{4} \tilde{w}_{4}\right)_{i, j, k-0,5}^{n}}{\Delta z} \Delta t+G_{p 4 w_{i, j, k}}^{n} \Delta t
$$

$\varphi_{1_{i, j, k}}^{n+1}=\varphi_{i, j, k}^{n} \frac{\rho_{1_{i, j, k}}^{n}}{\rho_{1_{i, j, k}}^{n+1}}-\frac{\left(\rho_{1} \varphi \tilde{u}_{1}\right)_{i+0,5, j, k}^{n}-\left(\rho_{1} \varphi \tilde{u}_{1}\right)_{i-0,5, j, k}^{n}}{\Delta x} \frac{\Delta t}{\rho_{1_{i, j, k}}^{n+1}}-$

$$
-\frac{\left(\rho_{1} \varphi \tilde{v}_{1}\right)_{i, j+0,5, k}^{n}-\left(\rho_{1} \varphi \tilde{v}_{1}\right)_{i, j-0,5, k}^{n}}{\Delta y} \frac{\Delta t}{\rho_{1_{i, j, k}^{n+1}}^{n+1}}-
$$

$$
-\frac{\left(\rho_{1} \varphi \tilde{w}_{1}\right)_{i, j, k+0,5}^{n}-\left(\rho_{1} \varphi \tilde{w}_{1}\right)_{i, j, k-0,5}^{n}}{\Delta z} \frac{\Delta t}{\rho_{1_{i, j, k}}^{n+1}}+
$$$$
+\left(\varphi_{\mathrm{B}_{i, j, k}}^{n} G_{g_{\mathrm{B}_{i, j, k}}}^{n}+\varphi_{w_{i, j, k}}^{n} G_{g w_{i, j, k}}^{n}\right) \frac{\Delta t}{\rho_{1_{i, j, k}}^{n+1}}
$$

$\varphi=\left(k, c_{\mathrm{p}}, \mu, \lambda, a\right)$

$$
\begin{aligned}
& \text { - уравнения сохранения импульса по } \\
& \text { осям координат }
\end{aligned}
$$

$$
u_{1_{i, j, k}}^{n+1}=\tilde{u}_{1_{i, j, k}}^{n} \frac{\rho_{i_{i, j, k}}^{n}}{\rho_{1_{i, j, k}}^{n+1}}-\frac{\left(\rho_{1} \tilde{u}_{1} \tilde{u}_{1}\right)_{i+0,5, j, k}^{n}-\left(\rho_{1} \tilde{u}_{1} \tilde{u}_{1}\right)_{i-0,5, j, k}^{n}}{\Delta x} \times
$$

$\times \frac{\Delta t}{\rho_{1_{i, j, k}}^{n+1}}-\frac{\left(\rho_{1} \tilde{u}_{1} \tilde{v}_{1}\right)_{i, j+0,5, k}^{n}-\left(\rho_{1} \tilde{u}_{1} \tilde{v}_{1}\right)_{i, j-0,5, k}^{n}}{\Delta y} \frac{\Delta t}{\rho_{1_{i, j, k}}^{n+1}}-$

$-\frac{\left(\rho_{1} \tilde{u}_{1} \tilde{w}_{1}\right)_{i, j, k+0,5}^{n}-\left(\rho_{1} \tilde{u}_{1} \tilde{w}_{1}\right)_{i, j, k-0,5}^{n}}{\Delta z} \frac{\Delta t}{\rho_{1, j, k}^{n+1}}-$

$-\left(\tau_{x_{i, j, k}}^{12^{n}}+\tau_{x_{i, j, k}}^{13^{n}}+\tau_{x_{i, j, k}}^{14^{n}}\right) \frac{\Delta t}{\rho_{1_{i, j, k}}^{n+1}}+$

$+\left(u_{\mathrm{B}_{i, j, k}}^{n} G_{g_{\mathrm{B}_{i}, j, k}}^{n}+u_{w_{i, j, k}}^{n} G_{g w_{i, j, k}}^{n}\right) \frac{\Delta t}{\rho_{1_{i, j, k}}^{n+1}}$ 


$$
\begin{aligned}
& u_{2_{i, j, k}}^{n+1}=\tilde{u}_{2_{i, j, k}}^{n} \frac{\rho_{2_{i, j, k}}^{n}}{\rho_{2_{i, j, k}}^{n+1}}-\frac{\left(\rho_{2} \tilde{u}_{2} \tilde{u}_{2}\right)_{i+0,5, j, k}^{n}-\left(\rho_{2} \tilde{u}_{2} \tilde{u}_{2}\right)_{i-0,5, j, k}^{n}}{\Delta x} \times \quad-\frac{\left(\rho_{2} J_{2} \tilde{w}_{2}\right)_{i, j, k+0,5}^{n}-\left(\rho_{2} J_{2} \tilde{w}_{2}\right)_{i, j, k-0,5}^{n}}{\Delta z} \frac{\Delta t}{\rho_{i_{i, j, k}}^{n+1}}+
\end{aligned}
$$

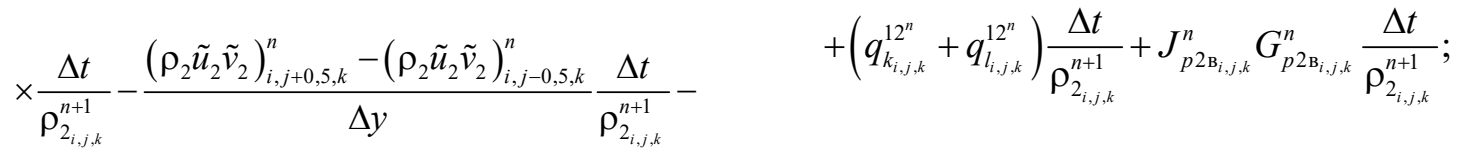

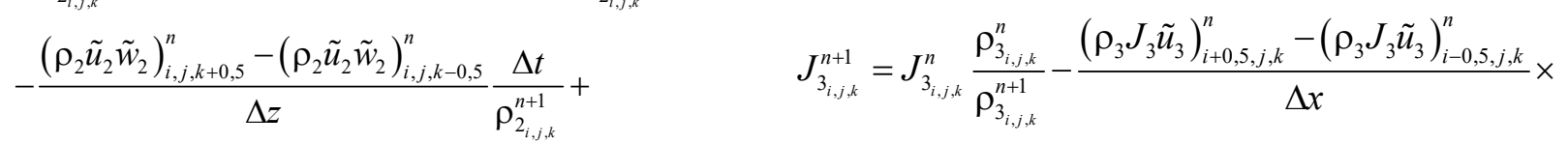

$$
\begin{aligned}
& +\tau_{x_{i, j, k}}^{12^{n}} \frac{\Delta t}{\rho_{2_{i, j, k}}^{n+1}}+u_{\mathrm{B}_{i, j, k}}^{n} G_{p 2 \mathrm{~B}_{i, j, k}}^{n} \frac{\Delta t}{\rho_{2_{i, j, k}}^{n+1}} \\
& u_{3_{i, j, k}}^{n+1}=\tilde{u}_{3_{i, j, k}}^{n} \frac{\rho_{3_{i, j, k}}^{n}}{\rho_{3_{i, j, k}}^{n+1}}-\frac{\left(\rho_{3} \tilde{u}_{3} \tilde{u}_{3}\right)_{i+0,5, j, k}^{n}-\left(\rho_{3} \tilde{u}_{3} \tilde{u}_{3}\right)_{i-0,5, j, k}^{n}}{\Delta x} \times-\frac{\left(\rho_{3} J_{3} \tilde{w}_{3}\right)_{i, j, k+0,5}^{n}-\left(\rho_{3} J_{3} \tilde{w}_{3}\right)_{i, j, k-0,5}^{n}}{\Delta z} \frac{\Delta t}{\rho_{3_{i, j, k}}^{n+1}}+
\end{aligned}
$$

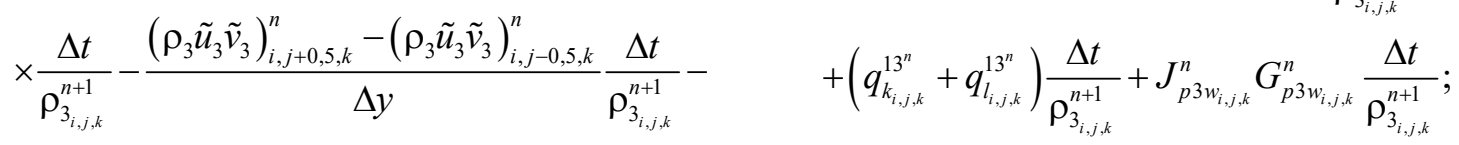

$$
\begin{aligned}
& -\frac{\left(\rho_{3} \tilde{u}_{3} \tilde{w}_{3}\right)_{i, j, k+0,5}^{n}-\left(\rho_{3} \tilde{u}_{3} \tilde{w}_{3}\right)_{i, j, k-0,5}^{n}}{\Delta z} \frac{\Delta t}{\rho_{3_{i, j, k}}^{n+1}}+\quad J_{4_{i, j, k}}^{n+1}=J_{4_{i, j, k}}^{n} \frac{\rho_{4_{i, j, k}}^{n}}{\rho_{4_{i, j, k}}^{n+1}}-\frac{\left(\rho_{4} J_{4} \tilde{u}_{4}\right)_{i+0,5, j, k}^{n}-\left(\rho_{4} J_{4} \tilde{u}_{4}\right)_{i-0,5, j, k}^{n} \times}{\Delta x} \times \\
& +\tau_{x_{i, j, k}}^{13^{n}} \frac{\Delta t}{\rho_{i_{i, j, k}}^{n+1}}+u_{w_{i, j, k}}^{n} G_{p 3 w_{i, j, k}}^{n} \frac{\Delta t}{\rho_{3_{i, j, k}}^{n+1}} ; \quad \times \frac{\Delta t}{\rho_{t_{i, j, k}}^{n+1}}-\frac{\left(\rho_{4} J_{4} \tilde{v}_{4}\right)_{i, j+0,5, k}^{n}-\left(\rho_{4} J_{4} \tilde{v}_{4}\right)_{i, j-0,5, k}^{n}}{\Delta y} \frac{\Delta t}{\rho_{3_{i, j, k}}^{n+1}}- \\
& u_{4_{i, j, k}}^{n+1}=\tilde{u}_{4_{i, j, k}}^{n} \frac{\rho_{4_{i, j, k}}^{n}}{\rho_{4_{i, j, k}}^{n+1}}-\frac{\left(\rho_{4} \tilde{u}_{4} \tilde{u}_{4}\right)_{i+0,5, j, k}^{n}-\left(\rho_{4} \tilde{u}_{4} \tilde{u}_{4}\right)_{i-0,5, j, k}^{n}}{\Delta x} \times-\frac{\left(\rho_{4} J_{4} \tilde{w}_{4}\right)_{i, j, k+0,5}^{n}-\left(\rho_{4} J_{4} \tilde{w}_{4}\right)_{i, j, k-0,5}^{n}}{\Delta z} \frac{\Delta t}{\rho_{4_{i, j, k}}^{n+1}}+
\end{aligned}
$$

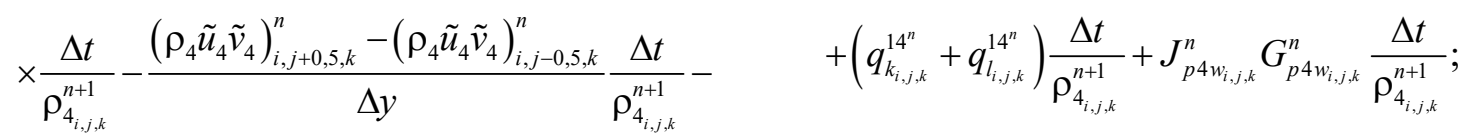

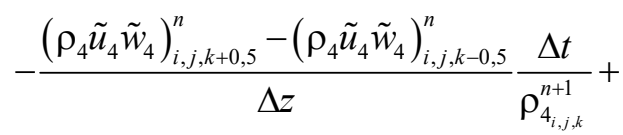

$$
\begin{aligned}
& +\tau_{x_{i, j, k}}^{14^{n}} \frac{\Delta t}{\rho_{4_{i, j, k}}^{n+1}}+u_{w_{i, j, k}}^{n} G_{p 4 w_{i, j, k}}^{n} \frac{\Delta t}{\rho_{4_{i, j, k}}^{n+1}} \\
& \text { - уравнения сохранения полной удельной } \\
& E_{1_{i, j, k}}^{n+1}=\tilde{E}_{1_{i, j, k}}^{n} \frac{\rho_{1_{i, j, k}}^{n}}{\rho_{1_{i, j, k}}^{n+1}}-\sum_{m=2}^{m=4}\left[\frac{\left(\rho_{m} E_{m}\right)_{i, j, k}^{n+1}-\left(\rho_{m} \tilde{E}_{m}\right)_{i, j, k}^{n}}{\rho_{1_{i, j, k}}^{n+1}}\right]-
\end{aligned}
$$

аналогично по другим направлениям $v_{l_{i, j, k}}^{n+1}, w_{l_{i, j, k}}^{n+1}$;

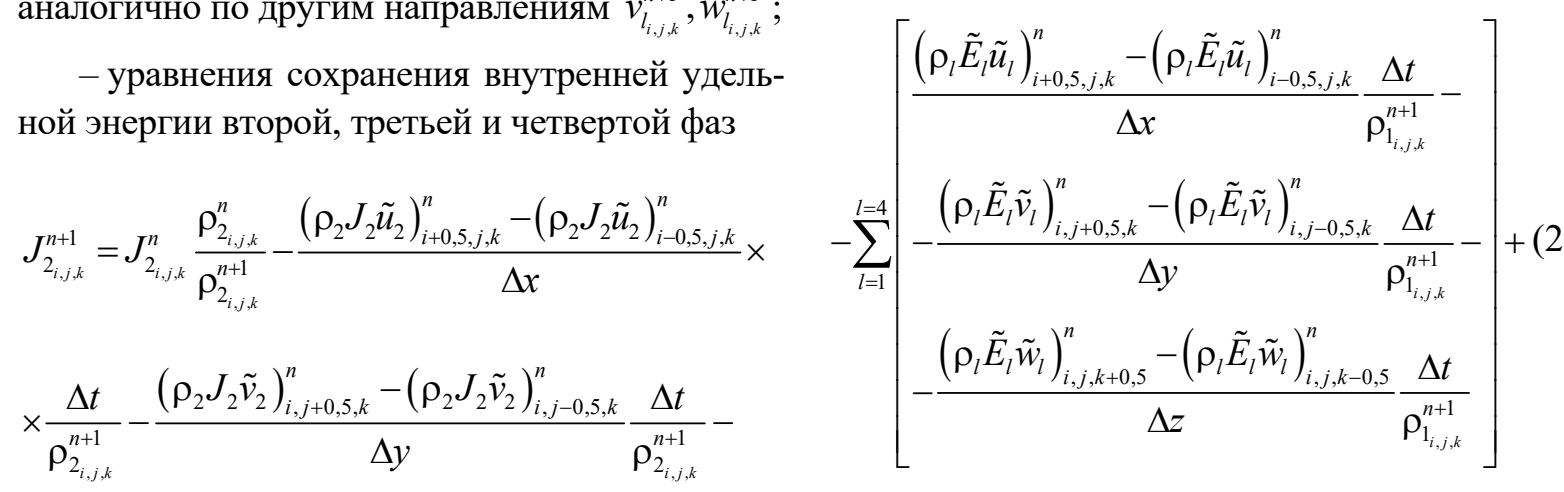




$$
\begin{aligned}
& +\left(\begin{array}{l}
E_{g \mathrm{~B}_{i, j, k}}^{n} G_{g \mathrm{~B}_{i, j, k}}^{n}+E_{g w_{i, j, k}}^{n} G_{g w_{i, j, k}}^{n}+E_{p 2 \mathrm{~B}_{i, j, k}}^{n} G_{p 2 \mathrm{~B}_{i, j, k}}^{n}+ \\
+E_{p 3 w_{i, j, k}}^{n} G_{p 3 w_{i, j, k}}^{n}+E_{p 4 w_{i, j, k}}^{n} G_{p 4 w_{i, j, k}}^{n}
\end{array}\right) \times \\
& \times \frac{\Delta t}{\rho_{1_{i, j, k}}^{n+1} .}
\end{aligned}
$$

Для повышения точности вычислений (обеспечения условия полной консервативности) в схему метода при расчете давления по уравнению состояния (15) вводится поправка, обеспечивающая (уточняющая) баланс по внутренней удельной энергии смеси [4].

Постановка граничных условий. Для описания граничных условий вокруг расчетной области (камера сгорания, сопловой блок и за сопловым блоком - см. рис. 1) вводятся слои фиктивных ячеек (рис. 2). На закрытых поверхностях (поверхность горения заряда смесевого твердого топлива, стенки камеры сгорания, соплового блока и заглушки соплового блока) используются условия непротекания: нормальная к поверхности компонента вектора скорости из приграничных ячеек сносится в слой фиктивных ячеек с противоположным знаком, остальные параметры потока из приграничных ячеек сносятся в слой фиктивных ячеек без изменения. На открытых поверхностях (расчетная область за сопловыми блоками) используется экстраполяция параметров потока. Приход продуктов сгорания от пиротехнического заряда воспламенительного устройства осуществляется в расчетные ячейки, в которых находится воспламенительное устройство. Приход продуктов сгорания с поверхности горения заряда смесевого твердого топлива осуществляется в расчетные ячейки, центры масс которых расположены в потоке у поверхности горения.

На нерегулярных (несовпадающих с координатной сеткой) криволинейных границах расчетной области применяется предложенный Ю.М. Давыдовым аппарат дробных ячеек (см. рис. 2). Здесь используется процедура нормального отображения фиктивной ячейки относительно границы расчетной области в поток. Везде применяются расчетные формулы только для целых ячеек.

Для вычисления скалярных газодинамических параметров граничной (фиктивной) ячейки, с учетом расщепления (параметры $\widetilde{\varphi}$ ), используются зависимости

$$
\begin{aligned}
& \varphi_{a}=\sum_{i}\left(V_{b_{i}} \varphi_{b_{i}}\right) ; \\
& \sum_{i} V_{b_{i}}=1 ; \varphi=\rho_{j}, k, \ldots, \lambda, a, \alpha_{j}, p, J_{j}, E_{j}, \\
& j=1 . . .4 .
\end{aligned}
$$

Для вычисления векторных газодинамических параметров граничной (фиктивной) ячейки, согласно условиям непротекания, с учетом расщепления (параметры $\widetilde{\varphi}$ ), используются зависимости:

$$
\begin{aligned}
& u_{a j}=\sum_{i}\left[V_{b_{i}}\left(-W n_{j} N_{x}+W n_{2 j} N_{x 2}\right)\right] ; \\
& v_{a j}=\sum_{i}\left[V_{b_{i}}\left(-W n_{j} N_{y}+W n_{2 j} N_{y 2}\right)\right] ; \\
& w_{a j}=\sum_{i}\left[V_{b_{i}}\left(-W n_{j} N_{z}+W n_{2 j} N_{z 2}\right)\right] ; \\
& \sum_{i} V_{b_{i}}=1, \quad j=1 \ldots 4 .
\end{aligned}
$$

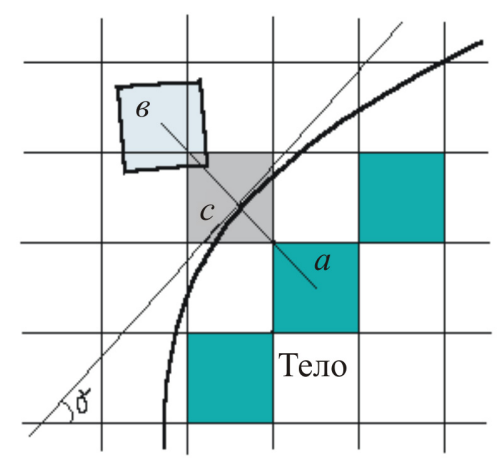

Рис. 2. Постановка граничных условий (схема)

В уравнениях (22), (23) приняты обозначения: $W n, W n_{1}, W n_{2}$ - проекции вектора скорости $\mathbf{W}$ на направляющие оси локальной системы координат $\left(W n_{1}=0\right) ; N_{x}, N_{y}, N_{z}-$ проекции нормали к плоскости отображения в базовой системе координат; $N_{x 2}, N_{y 2}, N_{z 2}$ - проекции оси локальной системы координат в базовой системе координат. Символы: $a-$ фиктивная ячейка, $b$ - отраженная фиктивная ячейка.

\section{Разгерметизация камеры сгорания}

В силу конструктивных особенностей соплового блока рассматриваемого РДТТ, имеющего заглушку в виде тонкой алюминиевой 
мембраны сферической формы, завальцованную в корпус сопла, вскрытие с непрогнозируемым разрушением на фрагменты и последующее движение фрагментов заглушки по каналу сопла рассматривать сложно. Ввиду этого разгерметизация камеры сгорания ракетного двигателя реализуется следующим упрощенным образом. До достижения определенного уровня давления продуктов сгорания в канале сопла (так называемого давления страгивания) заглушка сопла есть непроницаемая стенка. При превышении этого давления заглушка сопла мгновенно исчезает.

\section{Комплекс прикладных программ}

Для проведения численных расчетов на ЭВМ разработан комплекс прикладных программ, включающий в себя следующие основные модули:

- модуль расчета параметров, описывающих трехмерную постановку граничных условий на криволинейной образующей области интегрирования;

- основной расчетный модуль (main модуль) - расчет газодинамического течения в камере сгорания, сопловом блоке и за сопловым блоком РДТТ с учетом срабатывания воспламенительного устройства, прогрева, воспламенения и последующего горения заряда смесевого твердого топлива, разгерметизации камеры сгорания;

- модуль визуализации полученной расчетной информации.

Комплекс прикладных программ написан в среде программирования Kdevelop 4.6 для рабочей станции высокой производительности с операционной системой Linux Mint 17.3×86-64 на алгоритмическом языке $\mathrm{C} / \mathrm{C}++\mathrm{c}$ использованием (для основного расчетного модуля) стандарта многопотоковой обработки информации OpenCL [19-21]. Основная идея стандарта OpenCL состоит в реализации многопотокового выполнения кода по схеме «одна команда - много данных», т.е. одна операция одновременно применяется к большому массиву данных. Подобный подход позволяет в полной мере использовать вычислительный потенциал устройств с множеством относительно простых исполнительных модулей, например таких, как современные графические процессоры, и существенно (на порядок и более) повысить производительность вычислений.

\section{Проверка работоспособности программного продукта}

Проверка работоспособности программного продукта проводилась путем анализа вычислительной устойчивости и точности численного решения, оценки сходимости расчета на различных сетках, а также сравнения результатов расчета с данными стендовых испытаний РДТТ.

Наиболее критичной с точки зрения устойчивости и точности численного решения является задача газодинамического течения. Здесь для анализа свойств разностных схем метода Давыдова (см. выражения (16)-(21)) использовался эвристический подход, основанный на рассмотрении параболической формы их дифференциальных приближений $[4,14]$. В этом подходе оценивается знак коэффициентов диффузии $a_{i j}$ у диссипативных членов дифференциальных приближений, содержащих частные производные второго порядка по пространственным переменным. Эти коэффициенты обычно группируются в виде матрицы - так называемой матрицы аппроксимационной вязкости. Положительность детерминанта, либо диагональных элементов, либо следа матрицы аппроксимационной вязкости является условием вычислительной устойчивости исследуемой конечно-разностной схемы. Приближая значения этих параметров к нулю, повышаем точность вычислений.

Согласно трактовке лагранжева этапа метода Давыдова [14], каждая проекция конвективных членов исходной системы дифференциальных уравнений (10)-(13) на координатную ось может аппроксимироваться независимо. Это позволяет в рамках определенного приближения при анализе свойств разностной схемы ограничиваться одной координатой. Выпишем для одномерного однофазного аналога используемой выше конечно-разностной схемы метода (16)-(21) диагональные элементы матрицы аппроксимационной вязкости. Будем считать, что поток течет слева направо. Для противоположного направления потока достаточно поменять $\Delta x$ на $-\Delta x$. 
Итак, имеем:

$$
\begin{aligned}
& a_{11}=\frac{1}{2} u \Delta x-\frac{1}{4} u_{x} \Delta x^{2}+\frac{1}{2}\left(p_{\rho}-u^{2}\right) \Delta t ; \\
& a_{22}=\frac{1}{2} \rho u \Delta x-\frac{1}{2} \rho u_{x} \Delta x^{2}-\frac{1}{2}\left(\frac{3}{2}-\text { beta }\right) u \rho_{x} \Delta x^{2}+ \\
& +\frac{1}{2}\left(u p_{J J} J_{x}+u p_{J \rho} \rho_{x}+p_{J} u_{x}\right) \Delta x^{2}+ \\
& +\frac{1}{2}\left(-3 \rho u^{2}-\rho p_{\rho}-\frac{p}{\rho} p_{J}-u^{2} p_{J}\right) \Delta t ; \\
& a_{33}=\frac{1}{2} \rho u \Delta x-\frac{1}{4} \rho u_{x} \Delta x^{2}-\frac{1}{2}(1-\text { beta }) u \rho_{x} \Delta x^{2}- \\
& -\frac{1}{4} p_{J} u_{x} \Delta x^{2}-\frac{1}{2}\left(u p_{J J} J_{x}+u p_{\rho J} \rho_{x}\right) \Delta x^{2}+ \\
& +\frac{1}{2}\left[-\rho u^{2}+p_{J}\left(E-(1-2 \text { alfa }) \frac{p}{\rho}\right)\right] \Delta t \\
& \text { где } u_{x}=\frac{\partial u}{\partial x}, \quad p_{\rho J}=\frac{\partial^{2} p}{\partial \rho \partial J} \text { и т.д. }
\end{aligned}
$$

Условие положительности следа матрицы аппроксимационной вязкости $\left(a_{11}+a_{22}+a_{33}\right) \geq 0$ из выражений (24) рассматривалось в качестве критерия устойчивости выбранной конечно-разностной схемы метода.

Проводилась также оценка сходимости численного решения на различных по величине расчетной ячейки разностных сетках. Оценка сходимости показала, что для обеспечения требуемого уровня сходимости численного решения (на момент времени начала процесса срабатывания РДТТ) необходимо иметь в расчетной области 20 000000 расчетных ячеек.

Дополнительно проводилось сравнение результатов численного расчета с результатами натурных стендовых испытаний по изменению во времени давления в камере сгорания в районе переднего днища и тяги РДТТ. Результаты расчета и результаты стендовых испытаний хорошо согласуются между собой.

Работа выполнена при финансовой поддержке Российского фонда фундаментальных исследований и администрачии Пермского края (грант № 19-41-590006-р-а) и отмечена дипломом 1-й степени XXI Всероссийской НТК «Аэрокосмическая техника, высокие технологии и инновации - 2020».

\section{Библиографический список}

1. Соркин Р.Е. Газотермодинамика ракетных двигателей на твердом топливе. - М.: Наука, 1967. - 368 с.

2. Алемасов В.Е., Дрегалин А.Ф., Тишин А.П. Теория ракетных двигателей. - М.: Машиностроение, 1980. -533 с.

3. Численный эксперимент в теории РДТТ / А.М. Липанов, В.П. Бобрышев, А.В. Алиев [и др.]; под ред. А.М. Липанова; УИФ «Наука». - Екатеринбург, 1994. - 301 с.

4. Давыдов Ю.М., Егоров М.Ю. Численное моделирование нестационарных переходных процессов в активных и реактивных двигателях. - М.: НАПН, 1999. - 272 с.

5. Внутренняя баллистика РДТТ / А.В. Алиев, Г.Н. Амарантов [и др.]; под ред. А.М. Липанова и Ю.М. Милехина; РАРАН. - М.: Машиностроение, 2007. - 504 с.

6. Газовые течения в соплах энергоустановок / под ред. проф. В.Н. Емельянова. - М.: ФИЗМАТЛИТ, 2017. -328 c.

7. Энергетика и внутренняя баллистика ракетных двигателей на твердом топливе / Ю.М. Милехин, Г.В. Бурский, Г.С. Лавров, В.С. Попов, Д.Н. Садовничий. - М.: Наука, 2018. - 359 с.

8. Давыдов Ю.М., Давыдова И.М., Егоров М.Ю. Влияние полетной перегрузки на неустойчивость рабочего процесса в камере сгорания ракетного двигателя на твердом топливе // Доклады академии наук. 2004. - T. 398, № 2. - C. 194-197.

9. Davydov Yu.M., Davydova In.M., Egorov M.Yu. Flight overloading effect on the working process instability in the combustion chamber of a solid-fuel rocket engine // Doklady Physics. Moscow. Nauka / Interperiodica. - 2004. - Vol. 49, no. 9. - P. 527-529.

10. Численное моделирование внутрикамерных процессов при выходе на режим работы ракетного двигателя твердого топлива / Г.Н. Амарантов, М.Ю. Егоров, С.М. Егоров, Д.М. Егоров, В.И. Некрасов // Вычислительная механика сплошных сред. - 2010. - Т. 3, № 3. - С. 5-17.

11. Рихтмайер Р.Д., Мортон Х. Разностные методы решения краевых задач. - М.: Мир, 1972. - 420 с.

12. Рахматулин X.А. Основы газовой динамики взаимопроникающих движений сплошных сред // Прикладная математика и механика. - 1956. - Т. ХХ, № 2. - С. 184-195. 
13. Нигматулин Р.И. Динамика многофазных сред. - М.: Наука, 1987. - Ч. І. - 464 с.; ч. II. - 360 с.

14. Давыдов Ю.М. Крупных частиц метод // Математический энциклопедический словарь. - М.: Советская энциклопедия, 1988. - С. 303-304. (Математика. Большой энциклопедический словарь. - 2-е изд. М.: Российская энциклопедия, 1996. - С. 303-304; Математика. Большой энциклопедический словарь. 3-е изд. - М.: Российская энциклопедия, 1998/2000. - С. 303-304.)

15. Давыдов Ю.М., Давыдова И.М., Егоров М.Ю. Неустойчивость рабочего процесса в двухкамерном ракетном двигателе на твердом топливе // Доклады академии наук. - 2011. - Т. 439, № 2. - С. $188-191$.

16. Егоров М.Ю., Егоров Д.М. Численное исследование динамики внутрикамерных процессов при срабатывании бессоплового РДТТ // Известия вузов. Авиационная техника. - 2013. - № 1. - С. 51-54.

17. Егоров М.Ю. Численное исследование динамики внутрикамерных процессов при срабатывании специализированного РДТТ // Известия вузов. Авиационная техника. - 2017. - № 4. - С. 104-111.

18. Егоров М.Ю. Численное исследование динамики переходных внутрикамерных процессов при выходе на режим работы РДТТ особой компоновочной схемы // Известия вузов. Авиационная техника. 2019. - № 2. - С. 59-67.

19. Стахнов A.A. Linux. - СПб.: БХВ-Петербург, 2004. - 912 с.

20. Дерк Л. С и С++: справ. - М.: ВКК, 1997. - 592 с.

21. Programming Guide. AMD Accelerated Parallel Processing OpenCL. Advanced Micro Devices. 2011. -294 p.

\section{References}

1. Sorkin R.E. Gazotermodinamika raketnykh dvigateley na tverdom toplive [Gas-Thermodynamics of solid-fuel rocket engines]. Moscow: Nauka, 1967, 368 p.

2. Alemasov V.E., Dregalin A.F., Tishin A.P. Teoriya raketnykh dvigateley [Theory of rocket engines]. Moscow: Mashinostroyeniye, 1980, 533 p.

3. Lipanov A.M. et al. Chislennyy eksperiment v teorii RDTT [Numerical experiment in the theory of solid propellant motors]. Yekaterinburg: Nauka, 1994, $301 \mathrm{p}$.

4. Davydov Yu.M., Egorov M.Yu. Chislennoye modelirovaniye nestatsionarnykh perekhodnykh protsessov $\mathrm{v}$ aktivnykh i reaktivnykh dvigatelyakh [Numerical modeling of non-stationary transient processes in active and jet engines]. Moscow: NAPN, 1999, 272 p.

5. A.V. Aliyev and others. Vnutrennyaya ballistika RDTT [Internal ballistics solid propellant motors]. Ed. by A.M. Lipanov and Y.M. Milekhina. Moscow: Mashinostroyeniye, 2007, 504 p.

6. V.N. Yemelyanova. Gazovyye techeniya v soplakh energoustanovok [Gas flows in the nozzles of power plants]. Moscow: FIZMATLIT, 2017, 328 p.

7. Milekhin Yu.M., Burskiy G.V., Lavrov G.S., Popov V.S., Sadovnichiy D.N. Energetika i vnutrennyaya ballistika raketnykh dvigateley na tverdom toplive [Power Engineering and internal ballistics of rocket engines on solid fuel]. Moscow: Nauka, 2018, 359 p.

8. Davydov Yu.M., Davydova I.M., Egorov M.Yu. Vliyaniye polëtnoy peregruzki na neustoychivost rabochego protsessa $\mathrm{v}$ kamere sgoraniya raketnogo dvigatelya na tverdom toplive [The influence of flight overload on the instability of the working process in the combustion chamber of a solid-fuel rocket engine]. DOKLADY AKADEMII NAUK, 2004, vol. 398, No. 2, pp. 194-197.

9. Davydov Yu.M., Davydova In.M., Egorov M.Yu. Flight Overloading Effect on the Working Process Instability in the Combustion Chamber of a Solid-Fuel Rocket Engine. Doklady Physics. Moscow. Nauka / Interperiodica. 2004, no. 9, Vol. 49, pp. 527-529.

10. Amarantov G.N., Egorov M.Yu., Egorov S.M., Egorov D.M., Nekrasov V.I. Chislennoye modelirovaniye vnutrikamernykh protsessov pri vykhode na rezhim raboty raketnogo dvigatelya tvërdogo topliva [Numerical modeling of intra-chamber processes when entering the operating mode of a solid fuel rocket engine]. Computational continuum mechanics, 2010, vol. 3, no. 3, pp. 5-17.

11. RikhtmayYer R.D., Morton Kh. Raznostnyye metody resheniya krayevykh zadach [Difference methods for solving boundary value problems]. Moscow: Mir, 1972, 420 p.

12. Rakhmatulin Kh.A. Osnovy gazovoy dinamiki vzaimopronikayushchikh dvizheniy sploshnykh sred. PMM [Fundamentals of gas dynamics of interpenetrating motions of continuous media]. Journal of Applied Mathematics and Mechanics, 1956, vol. XX, no. 2, pp. 184-195.

13. Nigmatulin R.I. Dynamics of multiphase media. M.: Nauka, 1987, part I. 464 p.; part II. 360 p.

14. Davydov Yu. M. [Krupnykh chastits metod]. Large particle method. - In the book: Mathematical Encyclopedia. Moscow: Soviet Encyclopedia, 1988, pp. 303-304. Mathematics. A large encyclopedic dictionary. 
Ed-e 2-e-M: Russian Encyclopedia, 1996, p. 303-304. Mathematics. A large encyclopedic dictionary. Ed-e 3-e-M: Russian Encyclopedia, 1998/2000, pp. 303-304.

15. Davydov Yu.M., Davydova I.M., Egorov M.Yu. Neustoychivost rabochego protsessa v dvukhkamernom raketnom dvigatele na tverdom toplive [Instability of the working process in a two-chamber rocket engine on solid fuel]. Reports of the Russian Academy of Sciences, 2011, vol. 439, no. 2, pp. 188-191.

16. Egorov M.Yu., Egorov D.M. Chislennoye issledovaniye dinamiki vnutrikamernykh protsessov pri srabatyvanii bessoplovogo RDTT [Numerical study of the dynamics of intra-chamber processes in the event of a popleless solid fuel rocket engine]. Russian Aeronautics, 2013, no. 1, pp. 51-54.

17. Egorov M.Yu. Chislennoye issledovaniye dinamiki vnutrikamernykh protsessov pri srabatyvanii spetsializirovannogo RDTT [Numerical study of the dynamics of in-chamber processes when a specialized SRM is triggered]. Aviatsionnaya Tekhnika, 2017, no. 4, pp. 104-111.

18. Egorov M.Yu. Chislennoye issledovaniye dinamiki perekhodnykh vnutrikamernykh protsessov pri vykhode na rezhim raboty RDTT osoboy komponovochnoy skhemy [Numerical study of the dynamics of transient in-chamber processes when entering the operating mode of the special layout circuit RTTT]. Aviatsionnaya Tekhnika, 2019, no. 2, pp. 59-67.

19. Stahnov A.A Linux. St.Peterburg.: BKHV-Peterburg, 2004, 912 p.

20. Derk L. C and C++. Guide. Moscow: VKK, 1997, 592 p.

21. Programming Guide. AMD Accelerated Parallel Processing OpenCL. 2011. Advanced Micro Devices. 294 p.

\section{Об авторах}

Егоров Михаил Юрьевич (Пермь, Россия) - доктор физико-математических наук, профессор, профессор кафедры «Высшая математика» ФГБОУ ВО ПНИПУ (614990, г. Пермь, Комсомольский пр., д. 29, e-mail:_egorov-m-j@yandex.ru).

Егоров Дмитрий Михайлович (Пермь, Россия) - кандидат технических наук, первый заместитель генерального директора - главный конструктор АО НИИ полимерных материалов (614113, г. Пермь, ул. Чистопольская, д. 16, e-mail: egorovdimitriy@mail.ru).

Егоров Сергей Михайлович (Пермь, Россия) - кандидат физико-математических наук, начальник расчетного отдела АО НИИ полимерных материалов (614113, г. Пермь, ул. Чистопольская, д. 16, e-mail: know_nothing@bk.ru).

\section{About the authors}

Mikhail Yu. Egorov (Perm, Russian Federation) - Doctor of Physical and Mathematical Sciences, Professor of Higher Mathematics Department, Perm National Research Polytechnic University (29, Komsomolsky av., Perm, 614990, Russian Federation; e-mail: egorov-m-j@yandex.ru).

Dmitry M. Egorov (Perm, Russian Federation) - Candidate of Technical Sciences, First Deputy General Director - Chief Designer, JSC "Scientific-Research Institute of Polymeric Materials" (16, Chistopolskaya st., Perm, 614113, Russian Federation; e-mail: egorovdimitriy@mail.ru).

Sergey M. Egorov (Perm, Russian Federation) - Candidate of Physical and Mathematical Sciences, Head of the Calculation Department, JSC "Scientific-Research Institute of Polymeric Materials" (16, Chistopolskaya st., Perm, 614113, Russian Federation; e-mail: know_nothing@bk.ru).

Получено 16.02.2021 